\title{
mcr-1-Harboring Salmonella enterica Serovar Typhimurium Sequence Type 34 in Pigs, China
}

\section{Linxian Yi, ${ }^{1}$ Jing Wang, ${ }^{1}$ Yanling Gao, Yiyun Liu, Yohei Doi, Renjie Wu, Zhenling Zeng, Zisen Liang, Jian-Hua Liu}

We detected the mcr-1 gene in 21 (14.8\%) Salmonella isolates from pigs at slaughter; 19 were serovar Typhimurium sequence type 34 . The gene was located on IncHI2-like plasmids that also harbored IncF replicons and lacked a conjugative transfer region. These findings highlight the need to prevent further spread of colistin resistance in animals and humans.

Since our report of emergence of a plasmid-mediated co$\checkmark$ listin resistance mechanism involving the $\mathrm{mcr}-1$ gene in Escherichia coli (1), $m c r-1$ has been found in $>30$ countries in 5 continents in $<1$ year (2). E. coli is the main host of $m c r-1$, although several other Enterobacteriaceae, including Salmonella, have also been implicated as carriers of this gene $(3,4)$. We screened pigs at slaughter for Salmonella isolates to elucidate the epidemiology of $m c r-1$ in this major foodborne pathogen, which a serious public health problem.

\section{The Study}

During July 2013-May 2014, a total of 1,780 cecum samples were obtained from pigs at 5 slaughter houses in southern and central China. Samples were incubated in buffered peptone water for $20 \mathrm{~h}$ and then inoculated into chromogenic medium selective for Salmonella spp. (CHROMagar Microbiology, Paris, France) for 24 h. Suspected Salmonella colonies were selected ( 1 isolate was selected from each sample) and identified by using PCR for detection of the invA gene.

We obtained 142 Salmonella isolates and screened them for $m c r-1$ by using PCR and sequencing with primers described (1); 21 (14.8\%) were positive for $m c r-1$. These isolates were serotyped as described (5). We determined the clonal relationship of $m c r-1$-positive isolates by using pulsed-field gel electrophoresis (PFGE) with XbalI restriction enzyme and identified sequence types (STs) of these

Author affiliations: South China Agricultural University, Guangzhou, China (L. Yi, J. Wang, Y. Liu, R. Wu, Z. Zeng,

Z. Liang, J.-H. Liu); Henan General Institute of Animal Drugs and Feedstuff, Zhengzhou, China (Y. Gao); University of Pittsburgh Medical Center, Pittsburgh, Pennsylvania, USA (Y. Doi) isolates by using multilocus sequence typing (http://mlst. warwick.ac.uk/mlst/dbs/Senterica).

Most of the isolates had indistinguishable PFGE patterns and were clonally related to serovar Typhimurium ST34 (n = 19), which was the dominant type (Table). ST34 is common in isolates from humans in China and other countries and has been linked to food-producing animals $(6,7)$.

We determined MICs for 12 antimicrobial drugs for all $m c r$-1-positive isolates by using agar dilution methods or a broth microdilution method. Colistin showed MICs of $1-2 \mathrm{mg} / \mathrm{L}$ for these isolates (Table). More than $80 \%$ of the isolates were also resistant to ampicillin, streptomycin, florfenicol, tetracycline, sulfamethoxazole/trimethoprim, and gentamicin; $76.2 \%$ showed reduced susceptibility (MIC $>0.06 \mathrm{mg} / \mathrm{L}$ ) to ciprofloxacin.

We identified 2 lipopolysaccharide regulatory genes (pmrA and pmrB) by using and PCR and sequencing (8). Plasmid-mediated resistance genes floR (florfenicol resistance) and oqxAB (olaquindox and ciprofloxacin resistance) were detected by using PCR. None of the 21 isolates had mutations in the pmrA and $p m r B$ genes, which are associated with colistin resistance in Salmonella isolates. A total of 20 and 19 isolates had the floR and oqxAB genes, respectively. Salmonella Typhimurium ST34 has also been associated with the spread of the $o q x A B$ gene in human clinical isolates in China (9), and Salmonella Typhimurium ST34 coproducing oqxAB or floR genes has also been isolated from humans in Europe and Canada $(4,10)$.

To determine the genetic context of $m c r-1$, we used PCR mapping with primers ISAP-F (5'-CGAAGCACCAAGACATCA-3') and MCR-R (5'-CCACAAGAACAAA CGGACT-3'). Insertion sequence ISApll was found upstream of $m c r-1$ for all isolates.

We determined transferability and location of $m c r-1$ by using conjugation, transformation, and hybridization (with S1-PFGE nuclease). These procedures showed that $m c r-1$ genes were located on an $\approx 180$-kb plasmid, except for those in strains SH33 ( $\approx 110-\mathrm{kb}$ plasmid $)$ and SH239 $(\approx 180$-kb plasmid and $\approx 138$-kb plasmid) (Table). However, the $m c r-1$ gene could not be transferred to E. coli C600 by conjugation.

We then randomly selected $6 \mathrm{mcr}-1$-positive Salmonella isolates (SH138, SH149, SH175, SH36, SH39, and Z4P319S) for chemical transformation, which was successful for SH175, SH36, and Z4P319S. S1-PFGE con-

${ }^{1}$ These authors contributed equally to this article. 
Table. Characteristics of mcr-1-positive Salmonella isolates from pigs at slaughter, China, 2013-2014*

\begin{tabular}{|c|c|c|c|c|c|c|c|c|}
\hline Strain & Serovar & $\begin{array}{l}\text { PFGE } \\
\text { type }\end{array}$ & $\begin{array}{l}\text { Sequence } \\
\text { type }\end{array}$ & $\begin{array}{l}\text { S1-PFGE, } \\
\text { plasmid, kb }\end{array}$ & $\begin{array}{c}\text { Colistin } \\
\mathrm{MIC}, \mathrm{mg} / \mathrm{L}\end{array}$ & $\begin{array}{l}\text { Polymyxin B } \\
\text { MIC, mg/L }\end{array}$ & $\begin{array}{l}\text { Other antimicrobial } \\
\text { drug resistance } \dagger\end{array}$ & $\begin{array}{l}\text { Resistance } \\
\text { gene }\end{array}$ \\
\hline SH149 & Typhimurium & A5 & 34 & $\approx 180$ & 2 & 4 & $\begin{array}{l}\text { AMP, STR, GEN, } \\
\text { FFC, SXT, TET }\end{array}$ & floR, oqxAB \\
\hline SH143 & Typhimurium & A1 & 34 & $\approx 180$ & 2 & 4 & $\begin{array}{l}\text { AMP, STR, GEN, } \\
\text { FFC, SXT, TET }\end{array}$ & floR, oqxAB \\
\hline SH138 & Typhimurium & A1 & 34 & $\approx 180$ & 2 & 4 & $\begin{array}{l}\text { AMP, STR, GEN, } \\
\text { FFC, SXT, TET }\end{array}$ & floR, oqxAB \\
\hline $\mathrm{SH} 93$ & Typhimurium & $\mathrm{A} 1$ & 34 & $\approx 180$ & 2 & 8 & FFC, SXT, TET & floR, oqxAB \\
\hline SH33 & Typhimurium & A1 & 34 & $\approx 110$ & 2 & 4 & $\begin{array}{c}\text { AMP, STR, SXT, } \\
\text { TET }\end{array}$ & oqxAB \\
\hline SH137 & Typhimurium & $\mathrm{A} 1$ & 34 & $\approx 180$ & 2 & 8 & $\begin{array}{c}\text { AMP, STR, GEN, } \\
\text { FFC, SXT, TET }\end{array}$ & floR, oqxAB \\
\hline SA316 & Typhimurium & $\mathrm{A} 1$ & 34 & $\approx 180$ & 2 & 8 & $\begin{array}{l}\text { AMP, STR, GEN, } \\
\text { FFC, SXT, TET }\end{array}$ & floR \\
\hline SH127 & Typhimurium & A1 & 34 & $\approx 180$ & 2 & 4 & $\begin{array}{l}\text { AMP, STR, GEN, } \\
\text { FFC, SXT, TET }\end{array}$ & floR, oqxAB \\
\hline SH128 & Typhimurium & A1 & 34 & $\approx 180$ & 2 & 4 & $\begin{array}{l}\text { AMP, STR, GEN, } \\
\text { FFC, SXT, TET }\end{array}$ & floR, oqxAB \\
\hline SH133 & Typhimurium & A1 & 34 & $\approx 180$ & 2 & 4 & $\begin{array}{l}\text { AMP, STR, GEN, } \\
\text { FFC, SXT, TET }\end{array}$ & floR, oqxAB \\
\hline $\mathrm{SH} 134$ & Typhimurium & A1 & 34 & $\approx 180$ & 2 & 4 & $\begin{array}{l}\text { AMP, STR, GEN, } \\
\text { FFC, SXT, TET }\end{array}$ & floR, oqxAB \\
\hline $\mathrm{SH} 17$ & Typhimurium & $\mathrm{A} 1$ & 34 & $\approx 180$ & 2 & 4 & $\begin{array}{l}\text { AMP, STR, GEN, } \\
\text { FFC, SXT, TET }\end{array}$ & floR, oqxAB \\
\hline $\mathrm{SH} 239$ & Typhimurium & $\mathrm{A} 1$ & 34 & $\approx 180, \approx 138$ & 2 & 8 & $\begin{array}{l}\text { AMP, STR, GEN, } \\
\text { FFC, SXT, TET }\end{array}$ & floR \\
\hline $\mathrm{SH} 271$ & Typhimurium & $\mathrm{A} 1$ & 34 & $\approx 180$ & 2 & 8 & $\begin{array}{l}\text { AMP, STR, GEN, } \\
\text { FFC, SXT, TET }\end{array}$ & floR, oqxAB \\
\hline SH83 & Typhimurium & A1 & 34 & $\approx 180$ & 2 & 4 & $\begin{array}{l}\text { AMP, STR, GEN, } \\
\text { FFC, SXT, TET }\end{array}$ & floR, oqxAB \\
\hline $\mathrm{SH} 205$ & Typhimurium & A1 & 34 & $\approx 180$ & 2 & 8 & $\begin{array}{l}\text { AMP, STR, GEN, } \\
\text { FFC, SXT, TET }\end{array}$ & floR, oqxAB \\
\hline SH39 & Typhimurium & A2 & 34 & $\approx 180$ & 2 & 4 & $\begin{array}{l}\text { AMP, STR, GEN, } \\
\text { FFC, SXT, TET }\end{array}$ & floR, oqxAB \\
\hline SH178 & Typhimurium & A1 & 34 & $\approx 180$ & 2 & 4 & $\begin{array}{l}\text { AMP, STR, GEN, } \\
\text { FFC, SXT, TET }\end{array}$ & floR, oqxAB \\
\hline SH175§ & Typhimurium & A4 & 34 & $\approx 180$ & 2 & 8 & $\begin{array}{l}\text { AMP, STR, GEN, } \\
\text { FFC, SXT, TET }\end{array}$ & floR, oqxAB \\
\hline SH36§ & Heidelberg & B & ND & $\approx 180$ & 2 & 8 & STR, FFC, SXT & floR, oqxAB \\
\hline Z4P319S§ & London & C & ND & $\approx 180$ & 1 & 2 & $\begin{array}{l}\text { AMP, STR, GEN, } \\
\text { FFC,CIP, SXT, TET }\end{array}$ & floR, oqxAB \\
\hline
\end{tabular}

${ }^{*}$ According to recommendations of the joint Clinical and Laboratory Standards Institute (CLSI)-European Committee on Antimicrobial Susceptibility Testing (EUCAST) and the Polymyxin Breakpoints Working Group (http://www.eucast.org/), the International Organization for Standardization standard broth microdilution method (20776-1) was used to determine colistin MICs for these strains. Antimicrobial susceptibility was determined and evaluated according to CLSI document no. M100-S25 (http://shop.clsi.org/site/Sample_pdf/M100S25_sample.pdf). Resistance to florfenicol was interpreted according to EUCAST clinical breakpoints and epidemiological cutoff values ( $>16 \mathrm{mg} / \mathrm{L}$ ) (http://mic.eucast.org/Eucast2/). MICs of olaquindox for all isolates, except SH36, were >64 mg/L. AMP, ampicillin; CIP, ciprofloxacin; FFC, florfenicol; GEN, gentamicin; ND, not determined; STR, streptomycin; SXT, sulfamethoxazole/trimethoprim; TET, tetracycline.

†Boldface indicates resistance phenotypes transferred to a recipient by transformation.

‡Boldface indicates resistance genes transferred to a recipient by transformation.

§Original strains of transformants.

firmed that transformants harbored single plasmids and had 8-16-fold higher MICs for colistin than the recipient $E$. coli DH5 $\alpha$. The $f l o R$ and oqxAB genes were also transferred with the $m c r-1$ gene.

We used Hiseq Technology (Illumina, San Diego, CA, USA) to sequence plasmid DNA purified from transformants of Salmonella Heidelberg SH36 and Salmonella London Z4P319S and genomic DNA extracted from the original isolate (Salmonella Typhimurium SH138, which was selected as a representative ST34 strain). We assembled sequence reads were assembled into contigs by using SOAPdenovo version 2.04 (http://soap.genomics.org.cn/ soapdenovo.html) and the separated plasmid contigs of the $3 \mathrm{mcr}$-1-carrying plasmids from chromosomal contigs and compared them with pHNSHP45-2 by using blastn (http:// blast.ncbi.nlm.nih.gov/Blast.cgi) and BRIG (11). We then analyzed replicon sequence types of these plasmids by using the Plasmid MLST Database (http://pubmlst.org/plas$\mathrm{mid} /$ ) and performed analysis and annotation of the partial sequence of $m c r$-1-carrying plasmids by using the RAST Server (12), ISfinder (https://www-is.biotoul.fr), BLAST (http://blast.ncbi.nlm.nih.gov/Blast.cgi), and Gene Construction Kit 4.0 (Textco BioSoftware, Inc., West Lebanon, $\mathrm{NH}$, USA). 
A

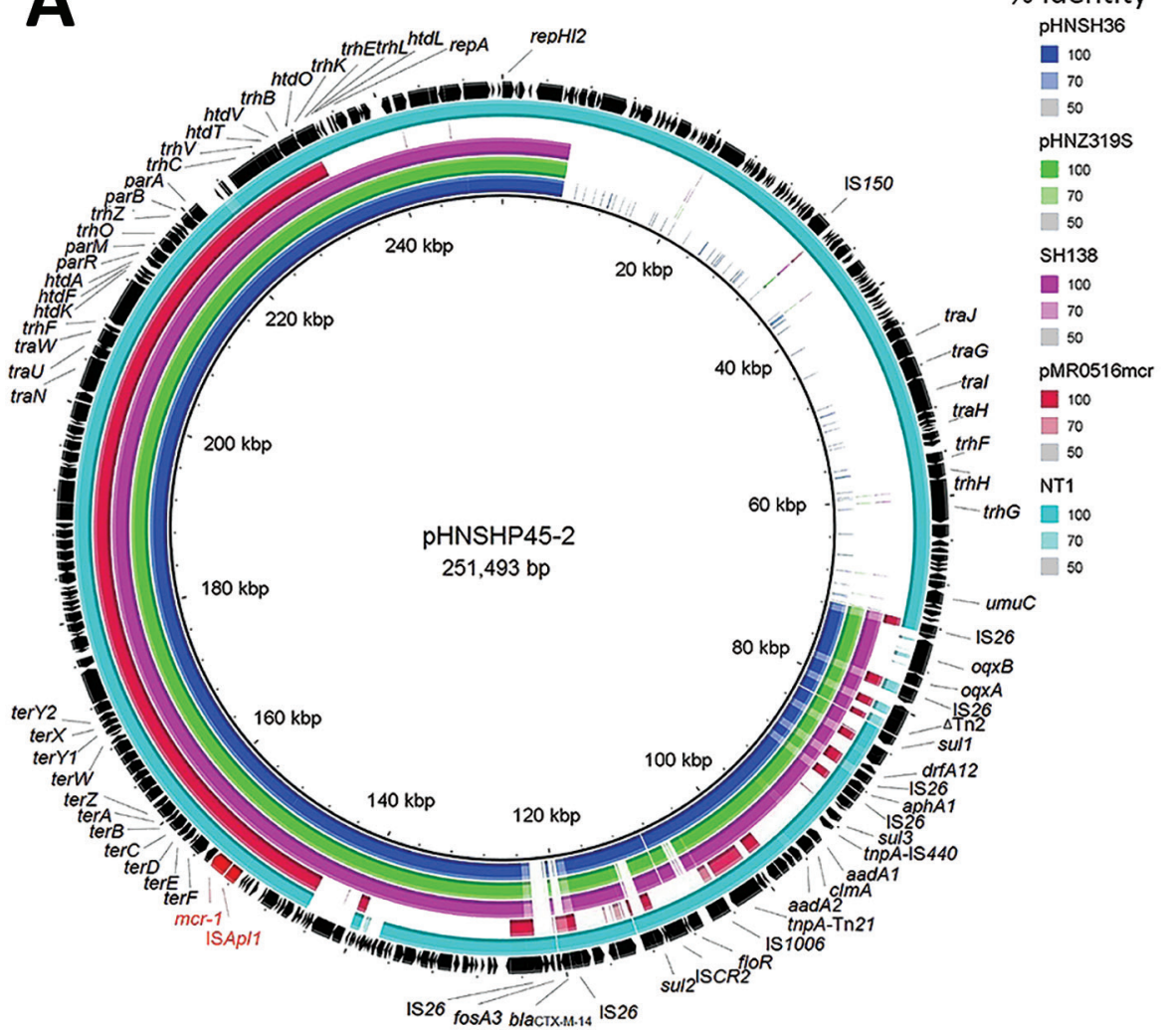

Figure 1. Sequence comparison of scaffolds (portions of genome sequences reconstructed from endsequenced whole-genome clones) identified in mcr-1-positive plasmids pHNSH36, pHNZ319S, and pHNSH138 with 2 mcr-1-bearing plasmids pHNSHP45-2 (GenBank accession no. KU341381) and pMR0516mcr (GenBank accession no. KX276657), and contigs identified in mcr-1-positive genomes of Escherichia coli strain NT1 in BRIG (11) (GenBank accession LSBW01000090.1) obtained during analysis of $m c r-1-$ positive Salmonella isolates from pigs at slaughter, China, 2013-2014. Arrows indicate positions and direction of transcription of genes. Reference plasmids pHNSHP45-2 (A) and pMR0516mcr (B) are indicated in black in the outer circles.

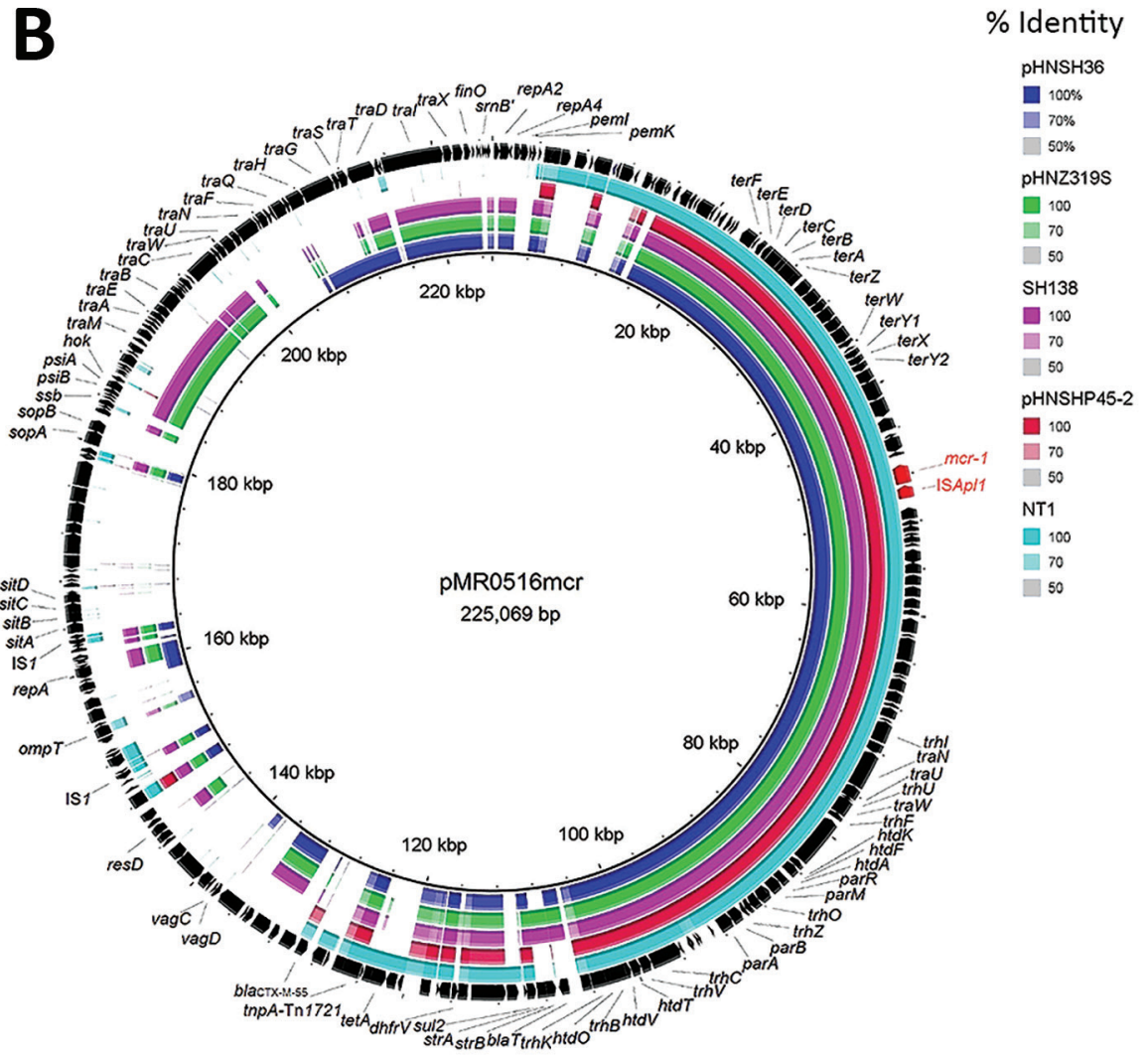


For the 3 isolates, we found $m c r-1$ on derivatives of IncHI2-like plasmids, which also harbored IncF (F4) and IncFIB (B53) replicons. The 3 plasmids were named pHNSH36 (from strain SH36), pHNZ319S (from strain Z4P319S), and pHNSH138 (from strain SH138). IncHI2 plasmids, such as pHNSHP45-2, have been frequently associated with global spread of $m c r-1$ genes $(3,13,14)$.

Compared with pHNSHP45-2 (in E. coli isolated from a pig in China [14]), the 3 plasmids all had a typical IncHI2-type backbone coding replication, transfer, maintenance, and stability functions, and a multiresistance region. However, we did not identify conjugative transfer region 1 of IncHI2 plasmids, including traJGIH

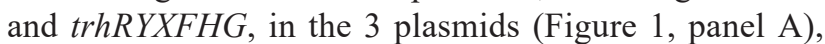
which might be the reason why these plasmids were not transferred by conjugation.

The multiresistance region contained numerous resistance genes, such as oqxAB, floR, sull, $c m l A$, aadA2, and complete or truncated insertion sequences and transposons (IS26, $\Delta \operatorname{Tn} 2, \operatorname{Tn} 21$, IS1006, and ISCR2), which was similar to that of pHNSHP45-2, except that pHNSH36, pHNZ319S, and $\mathrm{pHNSH} 138$ were missing fos $A 3$ and $b l a_{\text {CTX-M-14 }}$ (Figure 1, panel A). The 2,635-bp module (mcr-1- $\triangle \mathrm{IS} A p l 1)$ was similar to that of pHNSHP45-2, but different in some aspects, such as insertion sites and orientation, which were identical to those of the novel IncHI2-IncF plasmid pMR0516mer (GenBank accession no. KX276657) found in a clinical E. coli isolate from the United States (15), and human E. coli strain TN1 from Vietnam (GenBank accession no. LSBW01000090.1) (Figure 2).

Similar to pMR0516mcr (IncHI2-F18:A-:B1) (Figure 1, panel B), the 3 plasmids also contained an IncF-type backbone, including regions coding replication and partial regions coding transfer, maintenance, and stability functions. Similar to pHNSHP45-2, the plasmid carrying $m c r-1$ in strain NT1 belonged to the ST3-IncHI2 plasmid group. However, pMR0516mcr and the 3 plasmids described in this study were not typeable because there were no smr0199 loci in the IncHI2 plasmid (Figure 1, panel B). These data suggest that the $m c r-1$-ISApl1 module might have first been inserted into IncHI2 plasmids and that recombination between IncF- and IncHI2-type plasmids might have occurred subsequently, resulting in acquisition of the IncF-backbone fragment and deletion of the IncHI2 transfer region 1 or combination with the replication region in some instances.

\section{Conclusions}

We found that spread of $m c r-1$ in pigs at slaughter in China was associated with clonal dissemination of Salmonella Typhimurium ST34. This organism has also been detected in Portugal and England $(3,4)$. The presence of indistinguishable IncHI2-F4:A-:B5 plasmids in different Salmonella serovars indicates that horizontal transfer of $m c r-1-$ harboring plasmids might have also contributed to spread of $m c r-1$ in Salmonella spp.

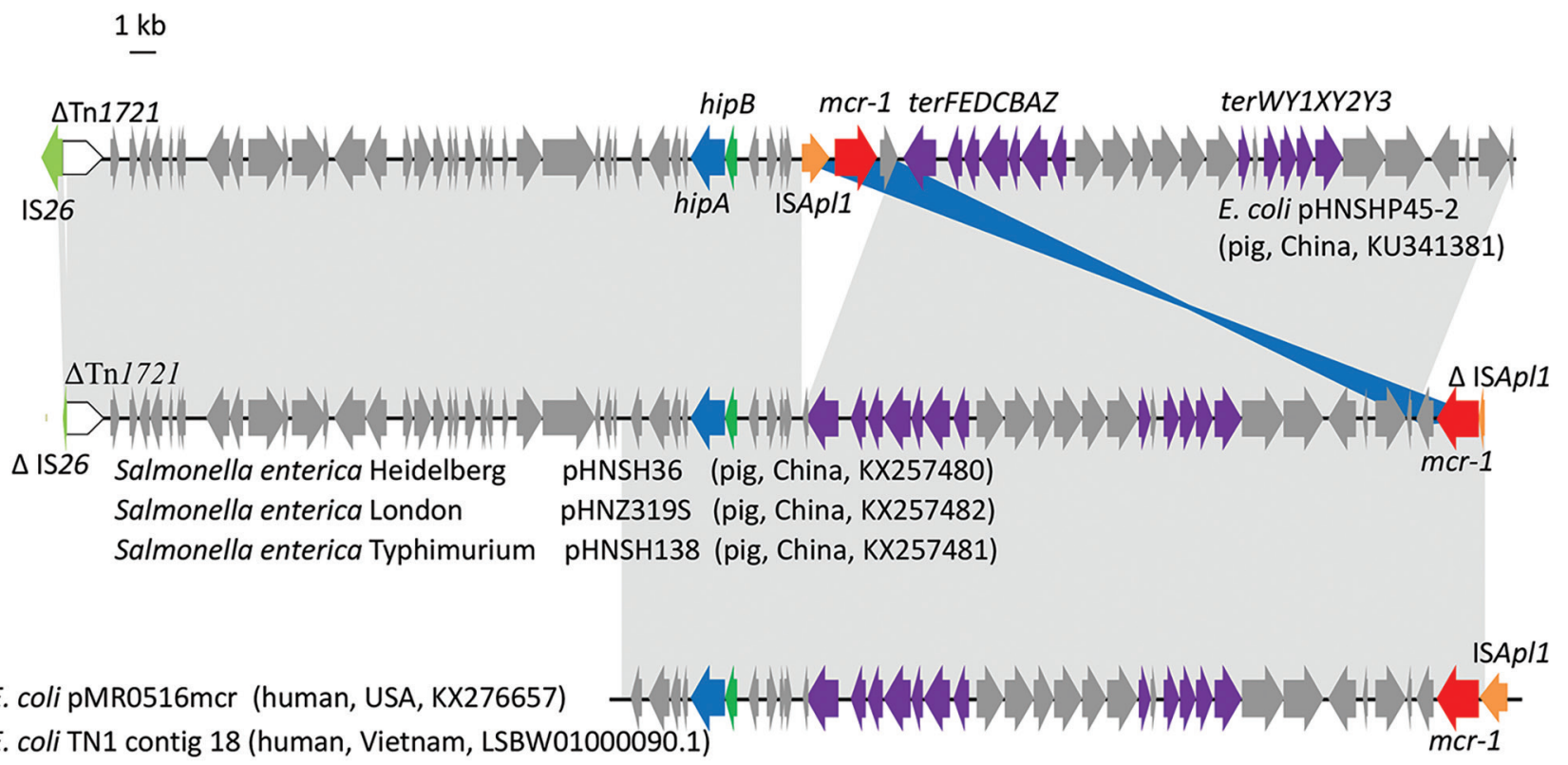

Figure 2. Genetic organization of scaffolds (portions of genome sequences reconstructed from end-sequenced whole-genome clones) containing mcr-1 harbored by plasmids pHNSH36, pHNZ319S, and pHNSH138 obtained during analysis of mcr-1-positive Salmonella isolates from pigs at slaughter, China, 2013-2014, and structural comparison with plasmids pHNSHP45-2, pMR0516mcr, and Escherichia coli TN1 contig 18. Arrows indicate positions and direction of transcription of genes. Regions with $>99 \%$ homology are indicated in gray or blue. Triangles indicate truncated genes. Information in parentheses after isolates indicate source, location, and GenBank accession number. 
Other drug-resistance genes, such as floR and oqxAB, were always transferred with $m c r-1$ by IncHI2-F4:A-:B5 plasmids, which led to successful flow of $m c r$-1-harboring Salmonella isolates under various selective pressures because florfenicol and olaquindox are widely used in farm animals in China. In addition, spread of similar IncHI2-like plasmids and Salmonella Typhimurium ST34 clones carrying $m c r-1$ in different countries highlights the need for internationally coordinated intervention strategies to limit its further dissemination.

This study was supported in part by grants from the National Key Basic Research Program of China (no. 2013CB127200) and the National Natural Science Foundation of China (no. 31272610 and no. 31625026).

Y.D. has served on advisory boards for Shionogi, Meiji Seika Pharma, Tetraphase Pharmaceuticals, and Merck, and has received research funding from Merck and The Medicines Company for studies not related to this study.

Ms. Yi is a researcher at the College of Veterinary Medicine, South China Agricultural University, Guangdong, China. Her primary research interest is microbiology, including antimicrobial resistance genes and their means of dissemination.

\section{References}

1. Liu YY, Wang Y, Walsh TR, Yi LX, Zhang R, Spencer J, et al. Emergence of plasmid-mediated colistin resistance mechanism MCR-1 in animals and human beings in China: a microbiological and molecular biological study. Lancet Infect Dis. 2016;16:161-8. http://dx.doi.org/10.1016/S1473-3099(15)00424-7

2. Schwarz S, Johnson AP. Transferable resistance to colistin: a new but old threat. J Antimicrob Chemother. 2016;71:2066-70. http://dx.doi.org/10.1093/jac/dkw274

3. Doumith M, Godbole G, Ashton P, Larkin L, Dallman T, Day M, et al. Detection of the plasmid-mediated $\mathrm{mcr}-1$ gene conferring colistin resistance in human and food isolates of Salmonella enterica and Escherichia coli in England and Wales. J Antimicrob Chemother. 2016;71:2300-5. http://dx.doi.org/10.1093/ jac/dkw093

4. Campos J, Cristino L, Peixe L, Antunes P. MCR-1 in multidrugresistant and copper-tolerant clinically relevant Salmonella 1,4,[5],12:i:- and $S$. Rissen clones in Portugal, 2011 to 2015. Euro Surveill. 2016;21:30270. http://dx.doi.org/10.2807/15607917.ES.2016.21.26.30270

5. Rahn K, De Grandis SA, Clarke RC, McEwen SA, Galán JE, Ginocchio C, et al. Amplification of an invA gene sequence of
Salmonella typhimurium by polymerase chain reaction as a specific method of detection of Salmonella. Mol Cell Probes. 1992;6:2719. http://dx.doi.org/10.1016/0890-8508(92)90002-F

6. Sun J, Ke B, Huang Y, He D, Li X, Liang Z, et al. The molecular epidemiological characteristics and genetic diversity of Salmonella Typhimurium in Guangdong, China, 2007-2011. PLoS One. 2014;9:e113145. http://dx.doi.org/10.1371/journal. pone. 0113145

7. Antunes P, Mourão J, Pestana N, Peixe L. Leakage of emerging clinically relevant multidrug-resistant Salmonella clones from pig farms. J Antimicrob Chemother. 2011;66:2028-32. http://dx.doi.org/10.1093/jac/dkr228

8. Quesada A, Porrero MC, Téllez S, Palomo G, García M, Domínguez L. Polymorphism of genes encoding PmrAB in colistin-resistant strains of Escherichia coli and Salmonella enterica isolated from poultry and swine. J Antimicrob Chemother. 2015;70:71-4. http://dx.doi.org/10.1093/jac/dku320

9. Wong MH, Yan M, Chan EW, Liu LZ, Kan B, Chen S. Expansion of Salmonella Typhimurium ST34 clone carrying multiple resistance determinants in China. Antimicrob Agents Chemother. 2013;57:4599-601. http://dx.doi.org/10.1128/ AAC.01174-13

10. Mulvey MR, Finley R, Allen V, Ang L, Bekal S, El Bailey S, et al. Emergence of multidrug-resistant Salmonella enterica serotype 4,[5],12:i:- involving human cases in Canada: results from the Canadian Integrated Program on Antimicrobial Resistance Surveillance (CIPARS), 2003-10. J Antimicrob Chemother. 2013;68:1982-6. http://dx.doi.org/10.1093/jac/dkt149

11. Alikhan NF, Petty NK, Ben Zakour NL, Beatson SA. BLAST Ring Image Generator (BRIG): simple prokaryote genome comparisons. BMC Genomics. 2011;12:402. http://dx.doi.org/10.1186/14712164-12-402

12. Aziz RK, Bartels D, Best AA, DeJongh M, Disz T, Edwards RA, et al. The RAST Server: rapid annotations using subsystems technology. BMC Genomics. 2008;9:75. http://dx.doi.org/ 10.1186/1471-2164-9-75

13. Zhi C, Lv L, Yu LF, Doi Y, Liu JH. Dissemination of the $m c r-1$ colistin resistance gene. Lancet Infect Dis. 2016;16:292-3. http://dx.doi.org/10.1016/S1473-3099(16)00063-3

14. Brennan E, Martins M, McCusker MP, Wang J, Alves BM, Hurley D, et al. Multidrug-resistant Escherichia coli in bovine animals, Europe. Emerg Infect Dis. 2016;22:1650-2. http://dx.doi.org/10.3201/eid2209.160140

15. McGann P, Snesrud E, Maybank R, Corey B, Ong AC, Clifford R, et al. Escherichia coli harboring $m c r-1$ and bla ${ }_{\text {СтХ-м }}$ on a novel IncF plasmid: first report of $m c r-1$ in the United States. Antimicrob Agents Chemother. 2016;60:4420-1. http://dx.doi.org/ 10.1128/AAC.01103-16

Address for correspondence: Jian-Hua Liu, College of Veterinary Medicine, South China Agricultural University, Guangzhou, Guangdong, China; email: jhliu@scau.edu.cn 